\title{
The PsyCLE project: Developing a psychology computer-based learning environment
}

\author{
NICK HAMMOND and JEAN MCKENDREE \\ University of York, York, England \\ and \\ PETER SCOTT \\ University of Sheffield, Sheffield, England
}

\begin{abstract}
PsyCLE is a 3-year project involving over 30 United Kingdom universities to develop multimedia resources for introductory psychology courses. Our goal is to support active learning and encourage students to form a sound understanding of concepts under study. The developments focus on interactive tasks, illustrative case study material, and tools to support critical thinking and analysis. We discuss the rationale for the approach adopted and describe the developed resources and their evaluation and use.
\end{abstract}

In 1992, the Higher Education Funding Councils in the United Kingdom announced an ambitious programthe Teaching and Learning Technology Programme (TLTP) - to promote use of technology in higher education so that it becomes an integral and established feature of the delivery of higher education. The program encouraged submissions from consortia of universities for developments to impact the maximum number of students. The 76 projects funded cover a wide variety of topics typical of university courses, with budgets ranging from about $\$ 100,000$ to $\$ 1,500,000$. Most of these bids involve consortia of from 2 to 40 sites. The Universities of York and Sheffield lead the psychology consortium, the PsyCLE project, with eight content modules covering auditory perception, visual perception, developmental psychology, social psychology, statistics, experimental design, memory, and philosophical roots, plus a generic concept-mapping module. We have matched each development site with 1 or more of our 31 evaluation sites.

\section{Rationale for the PsyCLE Developments}

Our aim is to develop support for introductory psychology courses that will yield significant added value over conventional teaching: we have no intention of providing an electronic textbook when a paper one would do a better job. Added value may be in terms of innovative approaches, perhaps supporting more flexible or more engaging learning activities, in terms of materials that cannot otherwise be provided, or in terms of greater flexibility in teaching, perhaps allowing lecturers to deploy their contact time with students more effectively. Different institutions, lecturers, and students have varied needs, so it

The work described in this paper was supported by a grant from the Higher Education Funding Council for England under the Teaching and Learning Technology Programme. Correspondence should be addressed to N. Hammond, CTI Centre for Psychology, University of York, Heslington, York Y01 5DD, England (e-mail: ctipsych@york.ac.uk). would be presumptuous of us to prescribe educational requirements and solutions; all we can do is point to what we see as the potential benefits of innovative approaches. Several factors that we have considered in the design of PsyCLE are discussed briefly below.

Making difficult topics more accessible. Some areas of psychology are difficult to convey through lectures or conventional printed material. We asked our developers, all psychology lecturers, to identify problem topics for which they considered use of computer presentation would bring benefits. Three examples of such topics, which are implemented in the developed materials, are attribution theory (in the social psychology module), retinal convolution (in the visual perception module), and basics of sound (in the auditory perception module). Thus in the retinal convolution materials, the student is able to simulate a set of nine "receptor cells" (for example, simulating what happens as an edge moves across the visual field) and to see how the underlying mathematics results in an excitatory or inhibitory response from the associated retinal ganglion cell. The student can switch back and forth between a textual description of the steps involved in convolution and the underlying mathematics, thus building up a more integrated understanding that combines interactive exploration, textual explanation, and computational representations.

A second example comes from the materials on the basics of sound. Here the student can change the frequency, amplitude, or phase of two sine waves and see the effect of adding the waves together. Any of the three waves (the two to be added and the resultant) can be heard by clicking on the waveform. As in the previous example, the computer supports multiple representations (waveforms, sounds, and a textual description) within an exploratory simulation.

For both of these examples, the interactivity of the computer would be hard to achieve by other means. It is likely, however, that the greatest benefit to students will accrue when use of the resources is integrated into wider 
course activities, perhaps part of an assignment, a laboratory practical class, or discussion in a small-group tutorial. It is still up to the lecturer to provide the most effective educational context for making use of the resources in the course.

Resources, not courses. If the developed materials are to be taken up in practice, it makes little sense to develop a fixed course for all introductory students. Mainstream psychology departments differ widely in the content and nature of their introductory courses, and psychology is also taught within the context of other disciplines, such as nursing or business studies. Even where the same material is covered, lecturers and departments will differ in approach and in how they choose to use computer-based materials. In some departments, the pressure may be to reduce the number of lectures and to encourage directed self-study; in others, the materials might be deployed to support tutorials or group assignments.

We have therefore attempted to design our materials so that they can support a range of learning activities for the student rather than conforming to a single educational approach. The retinal convolution materials described above, together with other facilities in the same module that allow students to explore simulations of experiments and processes in vision, is an example of a resource that can be used in a variety of teaching and learning situations.

A related issue is the importance of ensuring that, where feasible, the materials can be adapted by the lecturer for the requirements of the particular course. Gardner and Darby (1990) have suggested that the unwillingness of lecturers to make use of computer-based teaching materials is indicative of a not-invented-here syndrome that limits the spread of materials beyond their originating department. However, a survey conducted by Laurillard, Swift, and Darby (1993, p. 9) concluded that "the notinvented- here syndrome is no longer a potent force," and it seems more likely that the key factor is the difficulty of incorporating computer-based materials into the warp and weft of conventional educational activities. Courseware is seldom amenable to the process of seamless incorporation into the body of a lecturer's course in the way that conventional materials are, and all too often a software package must be used as it stands or be rejected. Our intention, therefore, was to allow the lecturer to edit, add to, or resequence materials. In practice, however, we have found it difficult to attain this goal of modifiability because of the technical and functional difficulties associated with a fully "open" development environment, and compromises have had to be made.

Supporting process as well as content. Our focus is to support learning activities as well as providing content materials. In part, learning support is embedded in the presentation of content, through interactive demonstrations, simulations, or case-based materials, but it can also be provided through generic tools and structures. The PsyCLE product consists of a mix of materials supporting both presentation and interactive learning activities, and we see this mix as essential not only to allow flexible usage, but also to provide the variety of educational activities necessary for deep learning.

One module, still under development, has the specific aim of helping students to think conceptually. The main aim of this module is to provide students with a facility to help them construct and reflect on conceptual structures. The facility is based on the technique of concept mapping, where learners are required to lay out their knowledge of a topic in a node-link structure (a concept map), with nodes denoting concepts, and links showing the relationships between these concepts. A screen illustrating the prototype concept-mapping facility available within PsyCLE is shown in Figure 1.

Concept maps can be constructed either on paper or using a computer-based concept-mapping tool. Conventional concept mapping, however, has a number of problems (see Reader \& Hammond, 1994a, 1994b), the main one being its generic nature: the technique provides no content-based schematic support either in terms of node and link types or syntax, or in terms of semantic content. We have therefore adapted the basic concept-mapping approach to make it more directed and sensitive to domain context by allowing the lecturer to constrain the map syntax to support only certain types of concepts and relationships. This provides learners with schematic scaffolding to help them relate concrete examples to the conceptual structures and arguments within the domain. Furthermore, providing schematic scaffolding leads to the possibility of defining a range of tasks with graded levels of support. The learner can be presented with a partial map to complete, such as a skeletal structure with no content-perhaps a set of labeled nodes to be organized into a structure, or even a completed but incorrect map to be reorganized. Each of these tasks provides some guidance but requires the student to "fill in the gaps," and a coherent set of tasks could be used to help the student focus on progressively more abstract or more advanced aspects of the topic to be learned.

The concept map in Figure 1 relates to the developmental psychology module on the topic of Piagetian stages of development. The tool bar on the left includes icons for the various node types available (theory, claim, and so forth); each of these node types is associated with different node icons. This prototype version of the tool also allows the student to associate weights with different nodes and links, thus providing a simple basis for evaluation of the evidence supporting or refuting different claims or theories.

Principles for effective learning from multimedia materials. Hypertext, hypermedia, and multimedia have received a good deal of "hype" within the computerbased learning fields. They certainly offer an exciting and creative way to deliver engaging, effective materials; but they also offer myriad opportunities for confusion and obfuscation. Multimedia designers have at times attempted to justify particular uses of this new technology through inappropriate reference to various psychological concepts. Elsewhere (McKendree, Reader, \& Hammond, 1995) we discuss three misconceptions concerning 


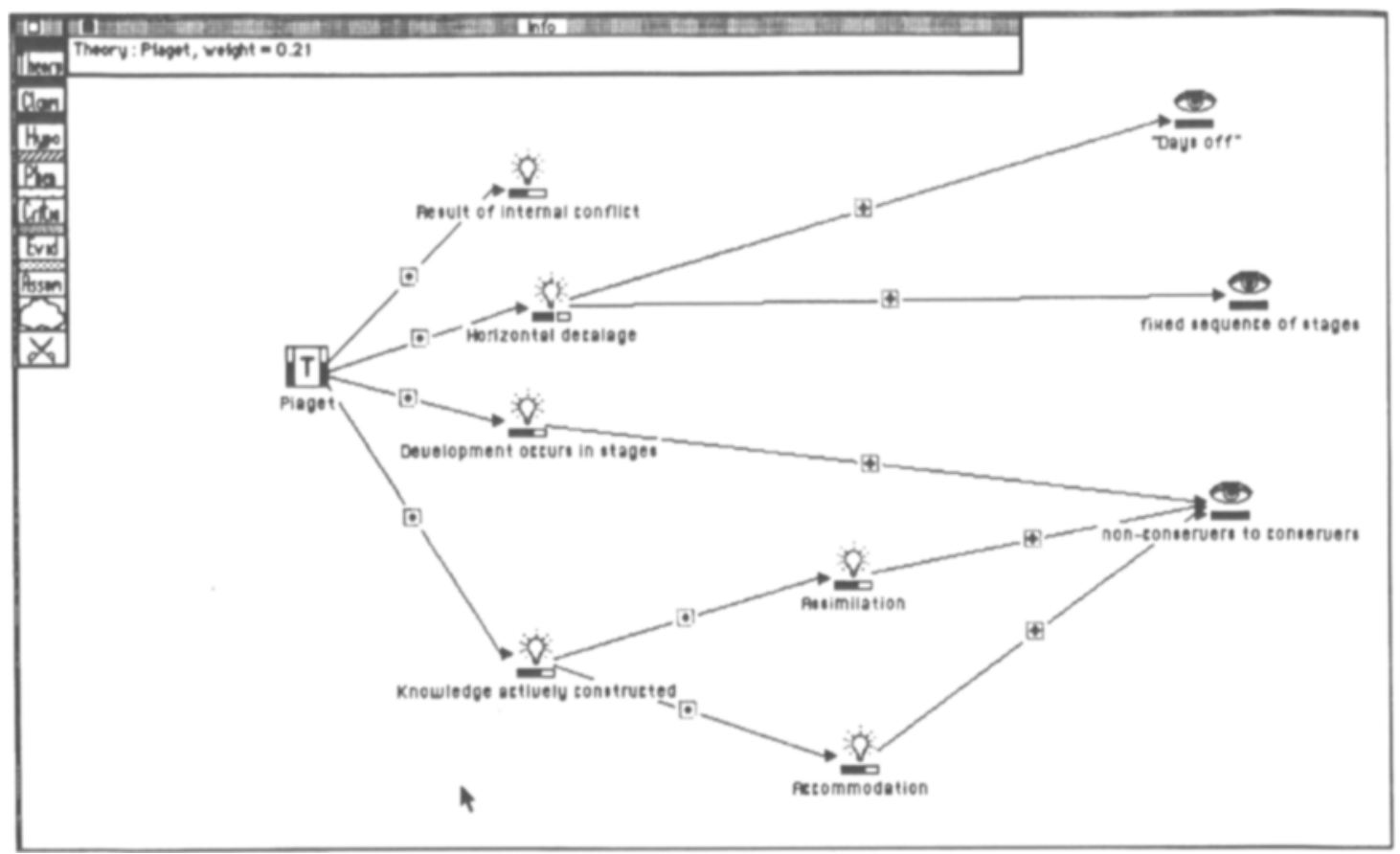

Figure 1. An example map from the concept-mapping module.

the use of hypermedia to support learning: that hypertext structures resemble the brain, that they resemble the structure of memory, and that these two resemblances underlie the claimed educational effectiveness of hypermedia. We have termed this the "homeopathic fallacy" of learning from hypermedia in analogy with the notion that the effects or appearance of a medicine must resemble the symptoms of the disease it is meant to treat.

Arguments that hypermedia is effective because "that's the way the mind is structured" are appealing because of their implied recommendations for design and the suggested simplicity of supporting learning. However, they fail to take account either of the importance of the educational context or of the view that conceptual learning results from directed task activity. We argue that it is not these supposed resemblances that make hypermedia potentially useful for learning, but the fact that it makes explicit the connections between chunks of information in the domain. The claim is that following the hypertext links gives the learner a clearer idea of how the information fits together in the domain to form a coherent whole. However, this is necessary but not sufficient: it is also contingent upon the learner processing the links in a meaningful way. Merely moving from one screen to another is unlikely to give the learner any insight about the implied connection between the two screens. This is compounded by the problem that the paths that the reader wishes to take, or that make sense to the reader, may not correspond with the author's view of the important connections. Unlike a book, which at least has familiar structures and genres, hypertext breaks down the predictability found in linear text. It is important, therefore, that hypermedia structures reflect narratives or thematic structures appropriate for learning, that they enable learners to stay "on task," and that they honor the overall learning goals.

Within PsyCLE, therefore, we have relied on four principles culled from educational and psychological theory: (1) use of concrete examples (abstraction is based on experience of multiple specific instances), (2) engagement (understanding results from meeting task goals through motivating learning activities), (3) appropriate guidance (learners need guidance to keep on track), and (4) learningby-doing (directed activity results in better engagement and memorization).

The first principle, use of concrete examples, is drawn from the view of learning termed situated cognition (Brown, Collins, \& Duguid, 1989), which emphasizes the importance of situating learning within the context of meaningful tasks. Using specific examples that relate to the learner's prior knowledge to new issues or to concepts being introduced provides one basis for concretization. In the PsyCLE developments, we have encouraged the use of simple experiments, with illustrations of readily grasped phenomena as a means for introducing a topic. Further, most modules start with one or more examples of readily grasped illustrations. For instance, the tutorial on nonverbal communication (from the social psychology module) starts with two short video clips illustrating social situations with contrasting degrees of nonverbal communication.

The principle of engagement refers to engagement with task or with conceptual understanding, not merely to engagement of mouse pointer with screen button. A multimedia system may provide a network of impressively packaged text, pictures, and sounds, but it is unlikely to engage the learner unless it provides structural 
support for learning. The system should encourage the learner to ask questions, to explore, and to form active hypotheses; in short, it should serve as a cognitive aid to learning (Hammond, 1993). This may not involve the delivery of any course materials at all; for example, the computer may help students in formulating their goals, in communicating with others, or in reflecting on and questioning their knowledge - hence the motivation for the concept-mapping facility.

The principles of appropriate guidance and of learningby-doing are by no means novel. Both are discussed more extensively in Hammond (1993) and underlie, for example, Carroll's (1990) approach to the training of computer skills, which is based on the conviction that you should immediately get the learner actively working on realistic tasks in the domain. Within PsyCLE, we have attempted to capitalize on the opportunities that use of the computer offers for activity-based learning.

\section{Evaluation and Future Directions}

We have described examples from several PsyCLE modules that, we hope, give a flavor of the approach we have adopted. Further examples can be seen in Hammond, McKendree, Reader, Trapp, and Scott (1995) and via the CTI Centre for Psychology World Wide Web site (url address: http://www.york.ac.uk/inst/ctipsych). Within the PsyCLE consortium, we have conducted formative evaluations of modules at critical stages of development: by subject experts at an early stage of specification and scenario definition; by students and lecturers with early prototypes, and by students and lecturers with $\beta$-test versions, where possible involving real teaching situations. In addition, all modules, and the approach in general, have been scrutinized by an expert advisory board. The early evaluations proved to be effective in alerting developers to shortcomings in the scope and content of the intended developments, in stimulating communication between developers and evaluators, and in helping the project as a whole identify the educational and technical criteria against which development should take place. Table 1 lists the criteria that were developed as part of the process of this early evaluation.

In regard to the evaluation of developed materials, feedback from experts, from lecturers, and from students has all been largely positive. As one might expect, the most critical comments tend to come from lecturers, and these are largely constructive. Our experience has been that the most effective procedure for fine-tuning the content and structure of the materials has been to conduct an in-depth "walk-through" session with a subject-matter expert, resulting both in the identification of critical problems and in recommendations and rationales for modification. In contrast, feedback from students is helpful in providing an overall "feel" and in identifying points of misunderstanding. In fact, the reported problems have often had more to do with the availability of machines and with organizational issues than with educational aspects.

The initial phase of the project was completed at the end of 1995. The project is continuing, albeit at a lower
Table 1

\section{PsyCLE Educational Criteria}

Learning process

Learning activities lead to improved understanding.

Learning activities result in better integration and memorization.

Learning activities motivate and encourage students.

Learning activities accommodate differences among students.

Appropriate variety of learnıng activities is provided.

Appropriate structures for learning are provided.

Learning outcomes

Quality of learning is improved.

Learning occurs more quickly.

Learning is more enjoyable.

Teaching criteria

Proposed change reduces the load on the lecturer.

Proposed change permits greater flexibility in teaching approach.

Proposed change supports more effective teaching strategies or activities.

Proposed change increases the motivation or enjoyment of the lecturer.

Proposed change encourages efficient reuse of materials.

Educational context criteria

Proposed change will be practicable within the typical institutional constraints.

Proposed change is simple to administer

Proposed change relates appropriately to concurrent learning or teaching activities.

Proposed change enhances other educational activities.

Proposed change allows appropriate monitoring of learning process and outcome.

Curriculum

Material is appropriate for introductory course.

Material is acceptable to most institutions.

Material has appropriate references or cross-links to related modules.

Material has a reasonable shelf life.

Quality of materials

Material is interesting.

Material is well structured or organized.

Material is well presented.

Tractability

Module can be implemented within the resources of the project. Module will run on standard delivery platforms.

level of funding, and will provide maintenance and support for the current materials and perhaps enable further development on a semicommercial basis. More generally, the PsyCLE project, and indeed the Teaching and Learning Technology Programme under which it is funded, can be seen as an experiment in a particular approach to the development of educational multimedia materials: development by academics within a large, nationwide consortium. The approach has of course attracted its fair share of criticism-for instance, in the problems of managing large distributed projects and the lack of involvement of expertise from the commercial field-but the final proof is in the developed product and how it is taken up in educational practice.

\section{REFERENCES}

Brown, J., Collins, A., \& Duguid, P. (1989). Situated cognition and the culture of learning. Educational Researcher, 18, 32-42.

CARroll, J. M. (1990). The Nurnberg funnel $\cdot$ Designing minimalist instruction for practical computer skill. Cambridge, MA: MIT Press. 
GARDNER, N., \& DARBY, J. (1990). Using computers in university teaching: A perspective on key issues. Computers \& Education, 15, 27-32.

Hammond, N. V. (1993). Learning with hypertext: Problems, principles and prospects. In C. McKnight, A. Dillon, \& J. Richardson (Eds.), Hypertext: A psychological perspective (pp. 51-69). Chichester, U.K.: Ellis Horwood.

Hammond, N. V., McKendree, J., Reader, W., Trapp, A., \& Scott, P. (1995, November). The PsyCLE project: Educational multimedia for conceptual understanding. In P. Zellweger (Ed.), Proceedings of Multimedia 95 (pp. 447-456). New York: ACM Press.

Laurillard, D., Swift, B., \& Darby, J. (1993). Academics' use of courseware materials: A survey. Association for Learning Technology Journal, 1, 4-14
MCKendree, J., Reader, W., \& Hammond, N. V. (1995). The "homeopathic fallacy" in learning from hypertext. Interactions, 2(3), 74-82.

READER, W. R., \& HAMMOND, N. V. (1994a). A comparison of structured and unstructured knowledge mapping tools in psychology teaching. In N. V. Hammond \& A. Trapp (Eds.), Proceedings of CiP 94 (pp. 57-58). York, U.K.: CTI Centre for Psychology.

Reader, W. R., \& Hammond, N. V. (1994b). Supporting learning from hypertext: Concept-mapping and beyond. Computers \& Education, 22, 99-106.

(Manuscript received November 13, 1995; revision accepted for publication January 31,1996 .) 